\title{
Vertical stiffness asymmetries during drop jumping are related to ankle stiffness asymmetries
}

Sean J. Maloney, Joanna Richards, Daniel G. D. Nixon, Lewis J. Harvey, lain M. Fletcher Institute of Sports Science and Physical Activity Research, University of Bedfordshire, Bedford, United Kingdom

Corresponding author

Sean Maloney, Research Graduate School, University of Bedfordshire, Polhill Avenue, Bedford, MK41 9EA

Sean.Maloney@beds.ac.uk +447800810099

Keywords

leg-spring behaviour, centre of mass displacement, spring-mass model, torsional spring model

Running head

Stiffness asymmetries in drop jumping

Acknowledgements

No benefits in any form have been or will be received from any commercial party or grant body related directly or indirectly in relation to this manuscript. The results of the current study do not constitute the endorsement of any product by the authors. 


\section{Abstract}

Asymmetry in vertical stiffness has been associated with increased injury incidence and impaired performance. The determinants of vertical stiffness asymmetry have not been previously investigated. Eighteen healthy males performed three unilateral drop jumps during which vertical stiffness and joint stiffness of the ankle and knee were calculated. Reactive strength index was also determined during the jumps using the ratio of flight time to ground contact time. 'Moderate' differences in vertical stiffness $\left(t_{17}=5.49 ; P<0.001\right)$, 'small' differences in centre of mass displacement $\left(t_{17}=-2.19\right.$; $P=0.043)$ and 'trivial' differences in ankle stiffness $\left(t_{17}=2.68 ; P=0.016\right)$ were observed between stiff and compliant limbs. A model including ankle stiffness and reactive strength index symmetry angles explained $79 \%$ of the variance in vertical stiffness asymmetry $\left(R^{2}=0.79 ; P<0.001\right)$. None of the symmetry angles were correlated to jump height or reactive strength index. Results suggest that asymmetries in ankle stiffness may play an important role in modulating vertical stiffness asymmetry in recreationally trained males. 


\section{Introduction}

Vertical stiffness aims to approximate the extent to which the leg compresses in response to ground reaction forces (McMahon \& Cheng, 1990) and, relative to other approximations of stiffness, is a quick and easy method by which to assess the viscoelastic properties of the lower limb (Butler et al., 2003). Given the proposed role of vertical stiffness in the modulation of injury risk and athletic performance (Butler et al., 2003; Pearson \& McMahon, 2012), the measurement of vertical stiffness is an important consideration for athletes and practitioners. Vertical stiffness is modifiable through both acute (Comyns et al., 2007; Moir et al., 2011) and chronic (Pearson \& McMahon, 2012) exercise interventions and the results of any assessment can therefore be used to directly inform the training process.

Vertical stiffness is based on the premise that the legs function as a global springmass system (Butler et al., 2003) and do not take into consideration how the ankle, knee and hip joints contribute to the summative stiffness of the overall system (Pearson \& McMahon, 2012). In order to elucidate the potential determinants of asymmetries in stiffness properties, it is important to consider the respective contribution of the stiffness of individual joints as well as vertical stiffness. Twodimensional computer simulation models created by Farley et al. (1998) and Farley and Morgenroth (1999) demonstrated that leg stiffness during bilateral hopping was modulated as a consequence of changes in ankle stiffness and was not affected by changes in knee stiffness. This proposition has been supported in hopping investigations by Kuitunen et al. (2011) and Kim et al. (2013) and in drop jumping by Arampatzis et al. (2001). Such a relationship would suggest that asymmetries in ankle stiffness are likely to lead to asymmetries in vertical stiffness. In contrast, Hobara et al. (2009) reported that knee stiffness, but not ankle stiffness, explained variance in 
vertical stiffness during maximal bilateral hopping. In addition, Horita et al. (2002) and Kuitunen et al. (2011) demonstrate that knee stiffness, whilst not affecting vertical stiffness, plays an important role in modulating mechanical output and overall performance. For these reasons, the role of the knee joint in modulating vertical stiffness asymmetries should not be discounted. To the authors' knowledge, no investigations have examined how asymmetries in joint stiffness may affect asymmetries in vertical stiffness.

Watsford et al. (2010) reported that pre-season asymmetries in vertical stiffness between Australian Rules football players that went on to sustain (asymmetry percentage: $7.3 \pm 6.1 \%)$ or not sustain $(7.4 \pm 5.7 \%)$ hamstring injuries were not significantly different. However, Watsford et al. (2010) did demonstrate that vertical leg stiffness of the affected limb in the injured group was significantly greater than the unaffected limb; no between-limb differences were observed in the non-injured group. A subsequent investigation from the same research group (Pruyn et al., 2012) found that mean vertical leg stiffness asymmetries recorded during the in-season competition period were higher in Australian rules footballers that experienced (asymmetry percentage: $7.5 \pm 3.0 \%$ ) lower body soft tissue injury than those that did not $(5.5 \pm 1.3 ; P<0.05)$. Given that several investigations have reported that asymmetries in force/power qualities may be detrimental to athletic performance (Bailey et al., 2013; Bazyler et al., 2014; Bell et al., 2014; Hart et al., 2014; Bailey et al., 2015), it may seem reasonable to hypothesise that asymmetries in leg stiffness may be similarly detrimental to performance as deformation of the lower limb will affect the transmission of force. Therefore, understanding the determinants of vertical stiffness asymmetries is likely to be an important factor for athletes and practitioners seeking to both reduce injury incidence and improve athletic performance. 
Stiffness of the ankle is likely to be of particular importance to the performance of stretch-shortening cycle activities such as drop jumping. Marshall et al. (2014) did not examine ankle stiffness, but correlated ankle power $(r=0.77 ; \mathrm{P}<0.01)$, plantar flexion moment $(r=0.65 ; \mathrm{P}<0.01)$ and ground contact time $(r=-0.48 ; \mathrm{P}=0.01)$ with faster change of direction test times. More specifically, Pruyn et al. (2014) reported significant relationships between stiffness of the musculature surrounding the ankle (medial gastrocnemius: $R=0.53$, soleus: $R=0.47$; both $P<0.05$ ) and faster change of direction performance. Whilst the role of true 'ankle stiffness' in modulating athletic performance requires further investigation, it is clear the kinetics and kinematics of the ankle joint are of particular importance.

Sugiyama et al. (2014) compared asymmetries during single leg approach jumping in basketball and reported greater jump heights and shorter ground contact times on the dominant limb. The investigators found that the symmetry index for jump height was not significantly correlated to the symmetry index of running velocity $(r=0.38 ; P=$ $0.08)$ or contact time $(r=0.32 ; \mathrm{P}=0.11)$, but was positively correlated with angular velocity $(r=0.41 ; P<0.05)$ and various parameters of angular displacement $(r=0.41$ $0.52 ; P<0.05)$ of the ankle. The results of Sugiyama et al. (2014) not only highlight the importance of ankle kinematics in athletic performance, but suggest that asymmetries in ankle stiffness may negatively influence performance outcomes. Further research is required to examine the interaction between ankle stiffness and vertical stiffness asymmetry.

The aim of the current study was to ascertain the determinants of vertical stiffness asymmetry during unilateral drop jumping. Previous research has shown that asymmetries may be differently expressed in different types of performance task, for example, cyclic versus acyclic (Flanagan \& Harrison, 2007) and bilateral versus 
unilateral (Benjanuvatra et al., 2013). Indeed, it has been shown that the expression of vertical stiffness asymmetry is different in bilateral hopping, bilateral drop jumping and unilateral drop jumping (Maloney et al., 2015). The unilateral drop jump may be the most appropriate task by which to assess vertical stiffness asymmetry in athletes required to perform maximal ballistic movements, such as jumps and changes of direction, within their sport as this demonstrates the greatest correspondence to these types of activity. As it has been shown that ankle stiffness is a strong determinant of vertical stiffness during hopping and may also explain asymmetries in jumping performance, it was hypothesised that asymmetries in vertical stiffness would be strongly related to asymmetries in ankle stiffness. 


\section{Materials and method}

\section{Participants}

Eighteen healthy males (age: $22 \pm 4$ years; height: $1.80 \pm 0.08 \mathrm{~m}$; body mass: $81.7 \pm$ $14.9 \mathrm{~kg}$ ) volunteered to participate in the study. A minimum sample size of eighteen participants was determined from an a priori power analysis (G*Power 3.1, HeinrichHeine-Universität, Düsseldorf, Germany) based upon an estimated squared multiple correlation of 0.45 between vertical stiffness and joint stiffness asymmetry angles and a power of 0.8 (Beck, 2013). Participants were recreationally active (undertaking $\geq 2.5$ hours of physical activity per week), reported no previous (within the last 12 months) or present lower limb injury and provided informed consent to participate in the study. Full ethical approval was granted by [REMAINDER OF SENTENCE REMOVED FOR ANONYMOUS PEER REVIEW PURPOSE]. All procedures were conducted in accordance with the Declaration of Helsinki.

\section{Experimental trials}

A familiarisation session was performed seven days prior to the experimental trial; a pilot investigation within the same experimental cohort had indicated that a single familiarisation session was appropriate for unilateral drop jumping. The familiarisation session was a complete simulation of the experimental trial outlined below.

All trials were conducted at the same time of day (10:00 - 12:00) for each participant, to alleviate the effects of circadian rhythms. The testing laboratory was controlled at an ambient temperature of $25^{\circ} \mathrm{C}$. Participants were instructed to prepare for testing as they would for training; nutrition, hydration and sleep were not monitored. The execution of each experimental trial was monitored by a United Kingdom Strength and 
Conditioning Association accredited strength and conditioning coach to ensure for consistency of technique.

\section{Warm-up}

All participants completed the same warm-up procedure outlined in Table 1. The warm-up procedure consisted of 15 dynamic exercises progressing from low to high intensities and from generic to specific movement patterns.

\section{*** Table 1 Here ***}

A rest period of 60 seconds was prescribed between each of the exercises from the specific movement preparation phase of the warm-up; all other exercises were not prescribed with rest periods. A rest period of 180 seconds was prescribed between the termination of the warm-up and commencement of the testing protocol.

\section{Testing}

All drop jump assessments were performed on a force plate system (Kistler 9281, Kistler Instruments, Winterthur, Switzerland). The force plate measured $0.6 \mathrm{~m} \times 0.4 \mathrm{~m}$ and was set flush into the laboratory floor as per manufacturer guidelines. Kinetic data was sampled at $1000 \mathrm{~Hz}$ and saved with the use of the manufacturer supplied software (BioWare 3.24, Kistler, Winterthur, Switzerland) for later analysis.

\section{Drop jump testing}

Participants performed a total of three, unshod unilateral drop jumps for each limb; the order in which participants performed unilateral drop jumps was randomised and counterbalanced. Each repetition of the drop jump was separated by 60 seconds to facilitate recovery between efforts (Read \& Cisar, 2001). For the execution of the drop 
jumps, participants stepped off a $0.18 \mathrm{~m}$ box and performed a vertical jump immediately upon landing. Participants were instructed to: a) step, not jump, off the box, b) minimise ground contact time during the landing phase whilst attempting to jump as high as possible, and c) attempt to maintain an upright torso; the execution of every jump was monitored for consistency of technique. Trials would have been excluded if any of these criteria were not met. Also, trials would have been excluded if participants landed heel first and a distinctive double peak in the vertical force trace was observed. All trials met the required criteria. Participants were cued to imagine the floor as 'hot coals' and to keep their 'eyes up'. Jumps were performed with participants' hands placed behind their head in order not to obscure the reflective markers. Jumps were performed to a self-selected depth by the participants in an attempt to replicate how they would jump in a training scenario.

\section{Kinematic analysis}

Drop jumping trials were recorded in the sagittal plane using a high-speed video camera (Quintic High-Speed LIVE USB 2, Quintic Consultancy Ltd., Coventry, United Kingdom) recording at $100 \mathrm{~Hz}$. Reflective joint markers were placed on the distal head of the fifth metatarsal, distal aspect of the lateral malleolus, lateral collateral ligament of the knee at the tibiofemoral gap, greater trochanter and anterolateral point of $11^{\text {th }}$ rib on both left and right sides of the body. Left limb jumps were recorded with the participants' left side of the body facing the camera, right limb jumps were recorded with the participants' right side of the body facing the camera. Recordings were automatically digitised using manufacturer provided software (Quintic Biomechanics v21, Quintic Consultancy Ltd., Coventry, United Kingdom). Data were filtered using a Butterworth fourth-order zero-lag filter (cut-off frequency $20 \mathrm{~Hz}$ ). 
Kinetic analysis

\section{${ }^{* * *}$ Figure 1 Near Here ${ }^{* * *}$}

Instants of initial foot contact, take-off and landing were identified from the vertical ground reaction force of each trial (Figure 1); this was determined as the time-point at which a clear change in force ( $\geq 10 \mathrm{~N}$ ) was observed (Lloyd et al., 2009). Reactive strength index was determined as the ratio of flight time to ground contract time during the jump (Newton \& Dugan, 2002) - the reactive strength index is an indicator of an athlete's ability to utilise the stretch-shortening cycle (Flanagan \& Comyns, 2008) and may therefore be linked to stiffness (Butler et al., 2003).

Inverse dynamics was used to express acceleration, velocity and centre of mass displacement; this was determined from the vertical force trace using the principles and equations described by Blazevich (2007). The vertical velocity of the hip joint marker at the instant of ground contact was used as the initial value for integration. Net muscle moments were determined using a rigid linked segment model, anthropomorphic data and an inverse dynamics analysis using the procedures outlined in Winter (2009); the linked segment model was created using Dempster's body segment parameter data (Dempster, 1955).

\section{Stiffness}

Vertical stiffness was calculated as the ratio of peak vertical ground reaction force $(\mathrm{N})$ relative to peak centre of mass displacement $(\mathrm{m})$ during the initial ground contact phase (Farley et al., 1998; Farley \& Morgenroth, 1999); this was averaged over the three drop jumps. The force-displacement correlation coefficient of the landing phase of each trial was required to be $\geq 0.8$ in an effort to ensure the efficacy of the spring- 
mass model (Padua et al., 2005). As vertical stiffness is affected by body size these values were reported relative to body mass (Farley et al., 1993).

Torsional stiffness of the ankle, knee and hip joints were calculated as the ratio of the change in net muscle moment $(\mathrm{N})$ to joint angular displacement (rad) between the initial ground contact phase and instant of peak angular displacement (Farley et al., 1998; Farley \& Morgenroth, 1999); these were averaged over the three recorded drop jumps. Pilot testing indicated that the timing of peak vertical ground reaction forces occurred at the instant of peak joint moments and maximum joint flexions as previously observed by Kuitunen et al. (2011) and that moment-displacement correlation coefficients were $\geq 0.8$. However, the phase shift for the moment displacement curve of the hip was $>10 \%$ (Figure 2). This has been previously specified as exclusion criteria in bilateral hopping trials (Farley et al., 1998) and stiffness of the hip was therefore not calculated in the current study.

\section{${ }^{* * *}$ Figure 2 Near Here ${ }^{* * *}$}

\section{Reliability}

The inter-session coefficient of variation for vertical stiffness was established in a previous investigation using the same experimental cohort over three trials, following a single familiarisation session, a value of $8 \%$ was recorded. The intra-session coefficients of variation for vertical stiffness in the current study were $5.3 \%$ and $6.5 \%$ listed for the stiff and compliant limbs respectively, coefficients of variation for centre of mass displacement were $7.2 \%$ and $4.3 \%$. Intra-session coefficients of variation for joint stiffness were (listed respectively for the stiff and compliant limbs): $1.6 \%$ and $2.2 \%$ for the ankle, $2.6 \%$ and $4.7 \%$ for the knee, and $4.1 \%$ and $4.9 \%$ for the hip, coefficients 
of variation for joint angular displacement were $3.8 \%$ and $5.3 \%$ for the ankle, $5.5 \%$ and $7.0 \%$ for the knee, and $10.9 \%$ and $11.0 \%$ for the hip.

\section{Statistical analysis}

Limbs were categorised as either stiff or compliant based upon the average vertical stiffness values achieved across the three drop jumps, each of the variables for the stiff and compliant limbs were subsequently compared. Asymmetries were quantified using the symmetry angle, calculated using the procedures outlined by Zifchock et al. (2008).

Shapiro-Wilks tests were performed to assess for normality; all variables were considered to be normally distributed given an alpha level of $P>0.05$. Paired sample T-tests were performed to analyse differences between the stiff and compliant limbs. Pair-wise effect sizes (d) (Cohen, 1998) were also calculated and interpreted using the thresholds defined by Hopkins (2003) where: $<0.20=$ trivial, $0.20-0.59=$ small, 0.60-1.19 = moderate, $1.20-1.99=$ large, and $\geq 2=$ very large .

Separate and independent forward step-wise regression analyses were performed for vertical stiffness for the stiff and compliant limbs to determine the influence of joint stiffness, joint angular displacement and joint moments - a total of six variables. Two additional forward step-wise regression analyses were performed, the first to determine the influence of vertical ground reaction force, centre of mass displacement, joint stiffness, joint angular displacement, centre of mass displacement and reactive strength index asymmetry angles on the vertical stiffness asymmetry angle - a total of nine variables - and a second with vertical ground reaction force, centre of mass displacement excluded - a total of seven variables. 
Finally, to examine the effect of stiffness and jump height asymmetry angles on performance, a linear correlation analysis of these variables to mean jump height (calculated as an average of each participant's stiff and compliant limbs) was performed. As symmetry angle values may be negative or positive to reflect left or right side dominance, negative values were transformed to positive values prior to examining the relationship with performance in order to evaluate differences solely in the magnitude of asymmetry.

Statistical significance was set at an alpha level of $P \leq 0.05$ and all statistical procedures were conducted using the Statistical Package for the Social Sciences for Windows (v19.0; SPSS Inc., Chicago, USA). 


\section{Results}

\section{*** Table 2 Here ***}

Stiff versus compliant limbs

Vertical stiffness was significantly different between the stiff and compliant limbs, such that stiffness of the compliant limb was an average of $17.8 \%$ lower than the stiff limb and associated with a 'moderate' effect size (Table 2). Relative to the stiff limb, ankle stiffness was an average of $6.8 \%$ lower in the compliant limb although the effect size was 'trivial'. Centre of mass displacement was an average of $9.4 \%$ greater in the compliant limb; the effect size between limbs was 'small'. No other significant differences were observed between the stiff and compliant limbs, although differences in vertical ground reaction force (2.3\%), angular hip displacement (11.3\%) and reactive strength index (6.3\%) were associated with 'small' effect sizes.

Determinants of asymmetry

A model including centre of mass displacement and vertical ground reaction force explained $99 \%$ of vertical stiffness asymmetry $\left(R^{2}=0.99\right.$; adjusted $R^{2}=0.99 ; F_{(1,15)}=$ 638.36; $P<0.001$ ). A model including centre of mass displacement alone explained $90 \%$ of vertical stiffness asymmetry $\left(R^{2}=0.90\right.$; adjusted $R^{2}=0.90 ; F_{(1,15)}=147.17 ; P$ $<0.001)$.

When centre of mass displacement and vertical ground reaction force were excluded, regression analyses revealed that a model including ankle stiffness and reactive strength index symmetry angles explained $79 \%$ of the variance in vertical stiffness asymmetry angle $\left(R^{2}=0.79\right.$; adjusted $\left.R^{2}=0.76 ; F_{(1,15)}=27.41 ; P<0.001\right)$ (Table 3$)$. 
In the neither the stiff nor compliant limb did a stepwise regression model explain the variance in vertical stiffness when vertical ground reaction force and centre of mass displacement were excluded.

Asymmetry and performance

Jump height symmetry angle did not correlate to jump height $(r=-0.35 ; P=0.157)$, nor did the symmetry angles of vertical stiffness $(r=-0.08 ; P=0.741)$, ankle stiffness $(r=-0.28 ; P=0.260)$ or knee stiffness $(r=0.06 ; P=0.815)$. The symmetry angles of vertical stiffness $(r=-0.01 ; P=0.96)$, ankle stiffness $(r=0.21 ; P=0.403)$ or knee stiffness $(r=0.004 ; P=0.987)$ did not correlate to jump height asymmetry. 


\section{Discussion}

The current study sought to ascertain the determinants of asymmetry in vertical stiffness as this had not been previously investigated. A regression model including ankle stiffness and reactive strength index asymmetries predicted $79 \%$ of the variance in vertical stiffness asymmetry. In addition, significant differences in ankle stiffness, but not knee stiffness, were observed between the stiff and compliant limbs. These results suggest that stiffness at the ankle may play an important role in modulating vertical stiffness asymmetry in a recreationally active population.

\section{Magnitude of asymmetry}

Significant differences in vertical stiffness were reported between the stiff and compliant limbs in the current study and were associated with a 'moderate' effect size. This is in agreement with the findings of a previous study utilising unilateral drop jumping (Maloney et al., 2015) and the magnitude of asymmetry observed in the current study (17.8\%) is similar to that previously reported (15.1\%). Also in agreement with Maloney et al. (2015), these differences were also shown to be a function of centre of mass displacement as opposed to ground reaction forces. Asymmetry in vertical stiffness during unilateral hopping has been associated with an increased risk of softtissue injury in Australian Rules football by Pruyn et al. (2012); asymmetries recorded within an 'injured' group were greater than asymmetries recorded in a 'non-injured' group $(7.5+3.0 \%$ versus $5.5+1.3 \% ; P<0.05)$. However, Watsford et al. (2010) had previously reported no difference within a similar population (injured: $7.3 \pm 6.1 \%$, noninjured: $7.4 \pm 5.7 \%)$. Vertical stiffness has also been determined during unilateral hopping in two other investigations (Hobara et al., 2013; Joseph et al., 2013). Although Joseph et al. (2013) did not discuss asymmetry, their data demonstrates magnitudes 
of asymmetry of between $3 \%$ and $7 \%$ similar to the values reported by Pruyn et al. (2012). Hobara et al. (2013) report slighter larger asymmetries of $9 \%$ to $13 \%$. Whilst it may be expected for asymmetries to be augmented during acyclic movements such as drop jumping versus cyclic tasks such as hopping - for example Maloney et al. (2015) reported vertical stiffness asymmetries of $15.1 \%$ during unilateral drop jumping and $5.3 \%$ during bilateral hopping respectively - the magnitude of asymmetry reported in the current study is larger than has been previously reported elsewhere in the literature. As the current study sampled recreationally trained individuals it is recommended that future research attempts to evaluate such asymmetries in athletically trained populations. It has been previously demonstrated that asymmetries are likely to be linked to training status (Bazyler et al., 2014).

\section{Contribution of ankle stiffness}

Vertical stiffness calculations do not take into consideration how the ankle, knee and hip joints interact and contribute to the summative stiffness of the overall system (Pearson \& McMahon, 2012). Simulation models had previously determined that vertical stiffness was regulated by ankle stiffness and not by knee stiffness (Farley et al., 1998; Farley \& Morgenroth, 1999). This position has been supported in hopping investigations by Kuitunen et al. (2011) and Kim et al. (2013) and in drop jumping by Arampatzis et al. (2001). In contrast, Hobara et al. (2009) reported that knee stiffness, but not ankle stiffness, explained variance in vertical stiffness during maximal bilateral hopping. Given these observations it is not surprising that asymmetries in vertical stiffness appear closely linked to asymmetries in ankle stiffness.

In a fixed system with multiple springs, the least stiff joint would be expected to undergo the greatest angular displacement in response to a given force (Farley et al., 
1998; Kuitunen et al., 2011), and therefore provide the greatest contribution to the summative displacement of the leg spring (i.e. centre of mass displacement in the current study). During unilateral drop jumping, joint stiffness was lower at the ankle (564 vs $526 \mathrm{~N} . \mathrm{m} \mathrm{rad}^{-1}$ for the stiff and compliant limbs respectively) and greater at the knee (2171 vs 2099 N.m.rad ${ }^{-1}$ ); the ankle was therefore the least stiff spring within the system. In line with the least stiff spring theory, the greatest angular displacement was observed at the ankle ( $36 \mathrm{deg})$, followed by the knee ( $27 \mathrm{deg})$ and then the hip (stiff limb: 12 deg, compliant limb: 13 deg). The results of the current study support the notion that the least stiff joint will have the greatest influence on the overall stiffness of leg-spring system (Kuitunen et al., 2011) and, perhaps, bilateral asymmetries therein.

\section{Comparisons with previous literature}

Kuitunen et al. (2011) reported greater stiffness at the knee versus the ankle during bilateral hopping at an intensity equivalent to 7 times bodyweight (39 and 12 N.m.deg ${ }^{1}$ respectively); this compares to values of $\sim 36$ and $\sim 9.5 \mathrm{~N} . \mathrm{m} \cdot \mathrm{deg}^{-1}$ in the current study. Joseph et al. (2013) also observed greater stiffness at the knee than at the ankle during unilateral hopping, reporting values of $0.14-0.22 \mathrm{~N} \cdot \mathrm{m}_{\mathrm{kg}}{ }^{-1} \cdot \mathrm{deg}^{-1}$ for the ankle and $0.17-0.35$ N.m. kg ${ }^{-1} \cdot \mathrm{deg}^{-1}$ for the knee; this compares to values of $\sim 0.12$ and 0.45 N.m. kg ${ }^{-1} . \mathrm{deg}^{-1}$ in the current study. In contrast, stiffnesses of 766 and 631 N.m.rad ${ }^{-1}$ were reported by Farley and Morgenroth (1999) for the ankle and knee during maximal height hopping, suggesting a greater relative reliance on ankle stiffness during bilateral hopping than during unilateral drop jumping. A similar trend was also observed during bilateral and unilateral drop jumping by Wang and Peng (2014). When performing unilateral drop jumps from a height of $0.2 \mathrm{~m}$, Wang and Peng (2014) reported respective stiffness values of $0.28,0.23$ and $0.38 \mathrm{~N} . \mathrm{m}_{\mathrm{rad}}{ }^{-1}$ for the 
ankle, knee and hip when expressed relative to body mass and body height, their choice of unit does not permit direct comparison to the current study.

\section{Drop jumping versus hopping tasks}

The figures for knee stiffness reported in the current study exceed those observed during bilateral or unilateral hopping. This is a likely consequence of a larger peak moment at the knee and indicates an increased reliance on knee extension during unilateral drop jumping. During bilateral hopping, peak moments at the knee of $\sim 150$ N.m ${ }^{-1}$ were reported by Farley and Morgenroth (1999), although moments of up to 475 $\mathrm{N} . \mathrm{m}^{-1}$ were reported by Kuitunen et al. (2011) with greater hopping intensities. Larger peak knee moments of up to $9 \mathrm{~N} \cdot \mathrm{m}^{-1} \cdot \mathrm{kg}^{-1}$ were reported by Joseph et al. (2013) during bilateral hopping, this compares to values of $\sim 13 \mathrm{~N} \cdot \mathrm{m}^{-1} \cdot \mathrm{kg}^{-1}$ in the current study. As indicated by Kuitunen et al. (2011), adjustments in knee stiffness appear to be related to the intensity of the activity; whilst ankle stiffness may contribute more strongly to the maintenance of overall vertical stiffness, knee stiffness plays an important role for optimising torque output. Indeed, ankle stiffness has been demonstrated to remain relatively consistent across a range of hopping intensities (Kuitunen et al., 2011). The structure of the knee extensors in relation to the plantar flexors, such as greater muscle size, longer muscle fibres and shorter tendons, facilitates greater mechanical work at the knee versus the ankle (Alexander \& Ker, 1990). Whilst Horita et al. (2002) and Kuitunen et al. (2011) demonstrated that knee stiffness did not influence global stiffness during bilateral hopping, they did report that knee stiffness plays an important role in modulating mechanical output and overall performance.

The angular displacements reported in the current study were greater than has been observed for bilateral hopping, for example, Farley and Morgenroth (1999) report 
respective values of $0.447,0.223$ and 0.059 rad (equivalent to 26,13 and 3 deg) for the ankle, knee and hip during maximum height hopping and Kuitunen et al. (2011) reported a 18-33 deg displacement at the ankle and 6-12 deg displacement at the knee. Whilst greater angular displacement of the knee is observed during unilateral drop jumping in comparison to bilateral hopping, Joseph et al. (2013) have reported greater knee flexion during unilateral hopping (35-45 deg). Greater flexion of the knee at ground contact would be expected to be advantageous to torque output (Kawakami et al., 2002).

Farley et al. (1998) propose that, in comparison to the hip and knee, the moment arm of ground reaction force is largest at the ankle and would be expected to result in the greatest angular displacement. Although absolute ground reaction forces in the current study are comparable to the hopping values of Farley and Morgenroth (1999) (typically 2000-3000 N), the ground reaction forces experienced during unilateral drop jumping are directed through a single limb and would therefore be expected to result in greater angular displacement. Moreover, Kuitunen et al. (2011) demonstrated that displacement of the ankle significantly increased in line with increases in ground reaction forces. It would therefore appear that the increased stiffness of the knee is consequential of the increased ground reaction forces and peak joint moments inherent with unilateral drop jumping versus bilateral hopping and enhanced requirement for muscular force to contribute to the production of torque required to overcome these ground reaction forces.

\section{Asymmetry and performance}

Although it has not been properly established if asymmetry in vertical stiffness is detrimental to athletic performance, several studies have linked asymmetry in other 
force/power characteristics to impaired performance (Bailey et al., 2013; Bazyler et al., 2014; Bell et al., 2014; Hart et al., 2014; Bailey et al., 2015), typically in an athletic population. Given that an asymmetry in vertical stiffness would be expected to result in an imbalanced force application to each limb (Wilson et al., 1994), it may be reasonable to propose that an asymmetry would be similarly detrimental to performance. The results of the current study would appear to refute this hypothesis in recreationally trained males; stiffness asymmetry angles did not significantly correlate with overall jump performance (mean jump height). Further investigation would be required before any conclusions may be drawn as these relationships should be examined in different athletic populations.

Sugiyama et al. (2014) reported that asymmetry in jump height was positively correlated with asymmetry in kinematic parameters of the ankle such as angular velocity and displacement during an approach jump in basketball. The current study did not observe such a relationship. Relationships between asymmetries in the displacement or stiffness of any joint and the asymmetry in vertical stiffness were not reported, nor were relationships between asymmetries in the stiffness of any joint. In addition, there was no relationship between the angular displacement of any joint and the vertical displacement of the centre of mass.

\section{Reactive strength index}

With the regression model showing the significance of the reactive strength index in explaining vertical stiffness asymmetry, this demonstrates a potential assessment option for the field-based assessment of asymmetry without the use of force plates and motion capture. Reactive strength index is a function of the flight time and ground contract time during a drop jump (Newton \& Dugan, 2002), and may therefore be 
quickly assessed using a jump mat or even a smartphone application. Whilst this does not replace the direct assessment of stiffness, it may offer an alternative way to assess stiffness-related asymmetries and is worthy of further investigation. Given the established relationship between reactive strength index and determinants of athletic performance, such as change of direction speed (Young et al., 2015), this may prove a particularly time-efficacious assessment tool for athletes and coaches.

\section{Conclusion}

In conclusion, asymmetries in ankle stiffness and reactive strength index appear to explain vertical stiffness asymmetry in recreationally trained males. Significant differences in ankle stiffness were also observed between the stiff and compliant limbs although the effect size was 'trivial'. As such, asymmetries in ankle stiffness appear to play a role in determining asymmetries in vertical stiffness. 


\section{Perspectives}

The measurement and quantification of vertical stiffness and joint stiffness asymmetries would appear to be of important practical relevance to athletes and coaches given the apparent link between asymmetry and injury incidence, and hypothesised link between asymmetry and performance. This study provides evidence to suggest that the ankle may play an important role in determining vertical stiffness asymmetries, although is limited by the sampling of recreationally trained athletes. Further research is required to explore the effects of stiffness asymmetries in welltrained athletic populations before any strong recommendations can be made. 


\section{References}

Alexander RM, Ker RF. The architecture of leg muscles. In: Winters JM, Woo S-Y, eds. Multiple muscle systems: biomechanics and movement organization. New York: Springer-Verlag, 1990:568-577.

Arampatzis A, Schade F, Walsh M, Brüggemann GP. Influence of leg stiffness and its effect on myodynamic jumping performance. Journal of Electromyography and Kinesiology. 2001: 11: 355-364.

Bailey $\mathrm{C}$, Sato $\mathrm{K}$, Alexander R, Chiang $\mathrm{C}-\mathrm{Y}$, Stone $\mathrm{MH}$. Isometric force production symmetry and jumping performance in collegiate athletes. Journal of Trainology. 2013: 2: 1-5.

Bailey CA, Sato K, Burnett A, Stone MH. Force production asymmetry in male and female athletes of differing strength levels. International Journal of Sports Physiology and Performance. 2015: 10: 504-508.

Bazyler CD, Bailey CA, Chiang C-Y, Sato K, Stone MH. The effects of strength training on isometric force production symmetry in recreationally trained males. Journal of Trainology. 2014: 3: 6-10.

Beck TW. The importance of a priori sample size estimation in strength and conditioning research. Journal of Strength and Conditioning Research. 2013: 27: 2323-2337.

Bell DR, Sanfilippo JL, Binkley N, Heiderscheit BC. Lean mass asymmetry influences force and power asymmetry during jumping in collegiate athletes. Journal of Strength and Conditioning Research. 2014: 28: 884-891.

Benjanuvatra N, Lay BS, Alderson JA, Blanksby BA. Comparison of ground reaction force asymmetry in one- and two-legged countermovement jumps. Journal of Strength and Conditioning Research. 2013: 27: 2700-2707.

Blazevich AJ. Position, Velocity and Acceleration. In: Blazevich AJ, ed. Sports Biomechanics: The Basics: Optimising Human Performance. London, England: A \& C Black Publishers Ltd, 2007:1-14.

Butler RJ, Crowell III HP, Davis IM. Lower extremity stiffness: implications for performance and injury. Clinical Biomechanics. 2003: 18: 511-517.

Cohen J. Statistical power analysis for the behavioral sciences. 2nd ed. New Jersey: Lawrence Erlbaum 1998.

Comyns TM, Harrison AJ, Hennessy L, Jensen RL. Identifying the optimal resistive load for complex training in male rugby players. Sports Biomechanics. 2007: 6: 59-70. Dempster WT. Space requirements of the seated operator. Ohio: Wright-Patterson Air Force Base 1955.

Farley CT, Glasheen J, McMahon TA. Running springs: speed and animal size. The Journal of Experimental Biology. 1993: 185: 71-86.

Farley CT, Houdijk HHP, Van Strien C, Louie M. Mechanism of leg stiffness adjustment for hopping on surfaces of different stiffnesses. Journal of Applied Physiology. 1998: 85: 1044-1055.

Farley CT, Morgenroth DC. Leg stiffness primarily depends on ankle stiffness during human hopping. Journal of Biomechanics. 1999: 32: 267-273.

Flanagan EP, Comyns TM. The use of contact time and the reactive strength index to optimize fast stretch-shortening cycle training. Strength \& Conditioning Journal. 2008: 30: 32-38.

Flanagan EP, Harrison AJ. Muscle dynamics differences between legs in healthy adults. Journal of Strength and Conditioning Research. 2007: 21: 67-72. 
Hart NH, Nimphius S, Spiteri T, Newton RU. Leg strength and lean mass symmetry influences kicking performance in Austrailian football. Journal of Sports Science and Medicine. 2014: 13: 157-165.

Hobara $\mathrm{H}$, Inoue $\mathrm{K}$, Kanosue $\mathrm{K}$. Effect of hopping frequency on bilateral differences in leg stiffness. Journal of Applied Biomechanics. 2013: 29: 55-60.

Hobara H, Muraoka T, Omuro K, Gomi K, Sakamoto M, Inoue K, Kanosue K. Knee stiffness is a major determinant of leg stiffness during maximal hopping. Journal of Biomechanics. 2009: 42: 1768-1771.

Hopkins WG. A spreadsheet for analysis of straightforward controlled trials. Sportscience, 2003.

Horita T, Komi PV, Nicol C, Kyröläinen H. Interaction between pre-landing activities and stiffness regulation of the knee joint musculoskeletal system in the drop jump: implications to performance. European Journal of Applied Physiology. 2002: 88: 7684.

Joseph CW, Bradshaw EJ, Kemp J, Clark RA. The interday reliability of ankle, knee, leg, and vertical musculoskeletal stiffness during hopping and overground running. Journal of Applied Biomechanics. 2013: 29: 386-394.

Kawakami $\mathrm{Y}$, Kubo K, Kanehisa $\mathrm{H}$, Fukunaga $\mathrm{T}$. Effect of series elasticity on isokinetic torque-angle relationship in humans. European Journal of Applied Physiology. 2002: 87: 381-387.

Kim W, João F, Tan J, Mota P, Vleck V, Aguiar L, Veloso A. The natural shock absorption of the leg spring. Journal of Biomechanics. 2013: 46: 129-136.

Kuitunen S, Ogiso K, Komi PV. Leg and joint stiffness in human hopping. Scandinavian Journal of Medicine and Science in Sports. 2011: 21: e157-e167.

Lloyd RS, Oliver JL, Hughes MG, Williams CA. Reliability and validity of field-based measures of leg stiffness and reactive strength index in youths. Journal of Sports Sciences. 2009: 27: 1565-1573.

Maloney SJ, Fletcher IM, Richards J. A comparison of methods to determine bilateral asymmetries in vertical leg stiffness. Journal of Sports Sciences. 2015.

Marshall BM, Franklyn-Miller AD, King EA, Moran KA, Strike SC, Falvey ÉC. Biomechanical factors associated with time to complete a change of direction cutting maneuver. Journal of Strength and Conditioning Research. 2014: 28: 2845-2851.

McMahon TA, Cheng GC. The mechanics of running: how does stiffness couple with speed? Journal of Biomechanics. 1990: 23: 65-78.

Moir GL, Mergy D, Witmer CA, Davis SE. The acute effects of manipulating volume and load of back squats on countermovement vertical jump performance. Journal of Strength and Conditioning Research. 2011: 25: 1486-1491.

Newton RU, Dugan E. Application of strength diagnosis. Strength \& Conditioning Journal. 2002: 24: 50-59.

Padua DA, Arnold BL, Carcia CR, Granata KP. Gender differences in leg stiffness and stiffness recruitment strategy during two-legged hopping. Journal of Motor Behaviour. 2005: 37: 111-126.

Pearson SJ, McMahon J. Lower limb mechanical properties: determining factors and implications for performance. Sports Medicine. 2012: 42: 929-940.

Pruyn EC, Watsford M, Murphy A. The relationship between lower-body stiffness and dynamic performance. Applied Physiology, Nutrition, and Metabolism. 2014: 39: 11441150.

Pruyn EC, Watsford ML, Murphy AJ, Pine MJ, Spurrs RW, Cameron ML, Johnston RJ. Relationship between leg stiffness and lower body injuries in professional Australian football. Journal of Sports Sciences. 2012: 30: 71-78. 
Sugiyama T, Kameda M, Kageyama M, Kiba K, Kanehisa H, Maeda A. Asymmetry between the dominant and non-dominant legs in the kinematics of the lower extremities during a running single leg jump in collegiate basketball players. Journal of Sports Science and Medicine. 2014: 13.

Wang LI, Peng HT. Biomechanical comparisons of single- and double-legged drop jumps with changes in drop height. International Journal of Sports Medicine. 2014: 35: 522-527.

Watsford ML, Murphy AJ, McLachlan KA, Bryant AL, Cameron ML, Crossley KM, Makdissi M. A prospective study of the relationship between lower body stiffness and hamstring injury in professional Australian rules footballers. American Journal of Sports Medicine. 2010: 38: 2058-2064.

Wilson GJ, Murphy AJ, Pryor JF. Musculotendinous stiffness: Its relationship to eccentric, isometric, and concentric performance. Journal of Applied Physiology. 1994: 76: 2714-2719.

Winter DA. Kinetics: forces and moments of force. Biomechanics and Motor Control of Human Movement. 4th ed. New Jersey: John Wiley \& Sons, Inc., 2009:107-138.

Young WB, Miller IR, Talpey SW. Physical qualities predict change-of-direction speed but not defensive agility in Australian rules football. Journal of Strength and Conditioning Research. 2015: 29: 206-212.

Zifchock RA, Davis I, Higginson J, Royer T. The symmetry angle: A novel, robust method of quantifying asymmetry. Gait \& posture. 2008: 27: 622-627. 


\section{Table 1:}

The experimental warm-up protocol completed by the participants.

\begin{tabular}{|c|c|c|}
\hline Warm-Up Phase & Exercise & $\begin{array}{l}\text { Prescription } \\
\text { (sets x reps) }\end{array}$ \\
\hline \multirow{8}{*}{$\begin{array}{l}\text { Generic Movement } \\
\text { Preparation }\end{array}$} & Inchworm & $1 \times 6$ \\
\hline & Quadruped thoracic rotation & 1x6each \\
\hline & Push up to 'T' & $1 \times 6 e a c h$ \\
\hline & Supine glute bridge with abduction & $1 \times 12$ \\
\hline & Mountain climber & 1x6each \\
\hline & Squat thrust to squat & $1 \times 6$ \\
\hline & Squat to Stand & $1 \times 6$ \\
\hline & $\begin{array}{l}\text { Single leg, stiff-legged deadlift to } \\
\text { reverse lunge }\end{array}$ & 1x6each \\
\hline \multirow{4}{*}{$\begin{array}{l}\text { Plyometric / Stiffness } \\
\text { Preparation }\end{array}$} & Lateral step down & $1 \times 8 e a c h$ \\
\hline & Single leg calf raise & $1 \times 8 e a c h$ \\
\hline & Alternate leg ankling drill & 1x8each \\
\hline & Vertical countermovement jump & $1 \times 4$ \\
\hline \multirow{3}{*}{$\begin{array}{l}\text { Specific Movement } \\
\text { Preparation }\end{array}$} & Bilateral hopping & $1 \times 10$ \\
\hline & Bilateral drop jump (from $0.18 \mathrm{~m}$ ) & $1 \times 2$ \\
\hline & Unilateral drop jump (from $0.18 \mathrm{~m}$ ) & 1x2each \\
\hline
\end{tabular}




\section{Table 2:}

Differences in kinetic and kinematic variables between the stiff and compliant limbs during unilateral drop jumping.

\begin{tabular}{|c|c|c|c|c|c|}
\hline & Stiff limb & $\begin{array}{l}\text { Compliant } \\
\text { limb }\end{array}$ & $t_{17}$ & $d$ & $P$ \\
\hline \multicolumn{6}{|l|}{ Stiffness } \\
\hline $\mathrm{K}_{\text {vert }}\left(\right.$ N.m.kg $\left.{ }^{-1}\right)$ & $190 \pm 52$ & $156 \pm 44$ & 5.49 & 0.70 & $<0.001^{*}$ \\
\hline $\mathrm{K}_{\text {ankle }}\left(\mathrm{N} \cdot \mathrm{m} \cdot \mathrm{rad}^{-1}\right)$ & $564 \pm 230$ & $526 \pm 194$ & 2.68 & 0.18 & $0.016^{*}$ \\
\hline $\mathrm{K}_{\text {knee }}\left(\mathrm{N} \cdot \mathrm{m} \cdot \mathrm{rad}^{-1}\right)$ & $2171 \pm 539$ & $2099 \pm 559$ & 1.65 & 0.13 & 0.188 \\
\hline \multicolumn{6}{|l|}{ Forces / moments } \\
\hline GRF $\left(N \cdot k^{-1}\right)$ & $29.44 \pm 2.68$ & $28.77 \pm 2.58$ & 1.27 & 0.25 & 0.222 \\
\hline Mankle $\left(N \cdot m \cdot \mathrm{kg}^{-1}\right)$ & $4.04 \pm 0.69$ & $3.94 \pm 0.95$ & 0.43 & 0.11 & 0.670 \\
\hline$M_{\text {knee }}\left(N \cdot m \cdot k^{-1}\right)$ & $12.40 \pm 2.24$ & $12.01 \pm 3.47$ & 0.55 & 0.14 & 0.591 \\
\hline $\mathrm{M}_{\text {hip }}\left(\mathrm{N} \cdot \mathrm{m} \cdot \mathrm{kg}^{-1}\right)$ & $18.19 \pm 7.59$ & $19.71 \pm 7.97$ & -0.78 & -0.20 & 0.449 \\
\hline \multicolumn{6}{|l|}{ Displacement } \\
\hline Dcom (m) & $0.17 \pm 0.05$ & $0.19 \pm 0.05$ & -2.19 & -0.36 & $0.043^{*}$ \\
\hline$D_{\text {ankle }}(\mathrm{rad})$ & $0.63 \pm 0.11$ & $0.61 \pm 0.15$ & 0.42 & 0.11 & 0.677 \\
\hline$D_{\text {knee }}(\mathrm{rad})$ & $0.48 \pm 0.10$ & $0.47 \pm 0.13$ & 0.13 & 0.03 & 0.899 \\
\hline Dhip (rad) & $0.20 \pm 0.08$ & $0.22 \pm 0.10$ & -1.43 & -0.27 & 0.170 \\
\hline \multicolumn{6}{|l|}{ Temporal } \\
\hline $\begin{array}{l}\text { RSI (flight time / } \\
\text { GCT) }\end{array}$ & $1.049 \pm 0.177$ & $0.983 \pm 0.208$ & 1.73 & 0.34 & 0.259 \\
\hline GCT (s) & $0.297 \pm 0.029$ & $0.301 \pm 0.035$ & -5.13 & -0.14 & 0.616 \\
\hline Time to peak force (s) & $0.149 \pm 0.035$ & $0.156 \pm 0.040$ & -1.17 & -0.19 & 0.102 \\
\hline
\end{tabular}


moment, $M_{\text {hip }}=$ hip moment, Dcom = centre of mass displacement, $D_{\text {ankle }}=$ ankle angular displacement, $D_{\text {knee }}=$ knee angular displacement, $D_{\text {hip }}=$ hip angular displacement, $\mathrm{RSI}=$ reactive strength index, GCT = ground contact time.

* indicates a significant difference between stiff and compliant limbs $(P<0.05)$. 
Table 3:

Results of the step-wise regression analysis for vertical stiffness symmetry angle.

\begin{tabular}{|c|c|c|c|c|c|}
\hline Model & $b$ & SE-b & Beta & $t$ & $P$ \\
\hline \multicolumn{6}{|l|}{1} \\
\hline (Constant) & -0.010 & 0.010 & & -0.825 & 0.422 \\
\hline Kankle $\alpha(\%)$ & 1.592 & 0.344 & 0.757 & 4.632 & $<0.001^{*}$ \\
\hline \multicolumn{6}{|l|}{2} \\
\hline (Constant) & -0.008 & 0.009 & & -0.914 & 0.375 \\
\hline $\mathrm{K}_{\text {ankle }} \alpha(\%)$ & 1.299 & 0.263 & 0.617 & 4.939 & $<0.001^{*}$ \\
\hline $\mathrm{RSI} \alpha(\%)$ & 0.633 & 0.164 & 0.481 & 3.851 & $0.002^{*}$ \\
\hline \multicolumn{6}{|c|}{$\begin{array}{l}\text { Dependant variable was vertical stiffness symmetry angle }(\%) \text {. } \\
\text { SE-b = standard error of } b, K_{\text {ankle }}=\text { ankle stiffness, } \alpha=\text { symmetry angle, } \\
\text { RSI = reactive strength index. } \\
\text { Model } 1: R^{2}=0.57 \text {, adjusted } R^{2}=0.55, P<0.001 \\
\text { Model } 2: R^{2}=0.79 \text {, adjusted } R^{2}=0.76, P<0.001 \\
\text { * indicates } P<0.001 \text {. }\end{array}$} \\
\hline
\end{tabular}


Figure Legends:

Figure 1 - An example of the vertical force trace associated with the drop jumps.

Figure 2 - An example moment-displacement curve for the ankle, knee and hip of a single participant from the instant of ground contact to the last instant before toe-off. 